\title{
Tonometry: an overview
}

\author{
Parul Singh \\ Department of Ophthalmology, Veer Chandra Singh Garhwali Government Medical Science and Research \\ Institute, Srinagar Garhwal, Uttarakhand, India.
}

\section{Introduction}

The intra-ocular pressure is a fundamental parameter of ocular health and disease. The intra-ocular pressure (IOP) is determined by the balance between the amount of aqueous humor- that the eye makes and the ease with which it leaves the eye. Not only IOP is important in diagnosis and management of glaucomatous conditions but, its assessment is imperative in the post-operative management of corneal, lenticular and vitreoretinal diseases. Measurement of IOP is hence of vital importance in ophthalmic examination. The quantitative assessment of intraocular pressure is known as tonometry; and the instruments used for tonometry are known as tonometer. Each technique has inherent advantages and disadvantages, none is ideal. Here, we describe different types of tonometers used currently to measure IOP.

\section{Assessment of IOP:}

Direct method:-

Manometry- This is used as a laboratory technique to perform continuous pressure measurements overtime in cadaveric eyes of humans or experimental animals.

Procedure- A hollow needle is introduced into the anterior chamber and connected to a reservoir of isotonic fluid, which is raised just enough to prevent any loss of aqueous from the eye. The height of the fluid column is calibrated in centimeters of water or millimeters of mercury to reflect the IOP.

Limitations-

- It is not practical for routine examinations.

- If it is utilized in humans, it requires general anaesthesia, which itself alters IOP.

\section{Indirect Methods:-}

1. Applanation tonometers:

a) Goldmann applanation tonometer (GAT)

b) Perkin tonometer

c) Drager's tonometer

d) Non- contact tonometer

e) Ocular response analyzer

f) Tono-pen

\section{Indentation tonometery:}

a) Schiotz tonometer

\section{Rebound tonometery}

4. Pascal dynamic contour tonometery

5. Non-corneal and trans-palpebral tonometery- Diaton

6. OCT tonometery

\section{Applanation tonometers:}

a) Goldmann applanation tonometer (GAT)-

Principle: It is based on Imbert ficks law which says that for ideal sphere (perfectly spherical, dry, infinitely thin walled and perfectly flexible) the pressure inside the sphere is equal to force required to flatten/ applanate the surface divided by the area of flattening.

Procedure: Patient is positioned at the slit lamp. The cornea is anesthetized and tear film is stained with $0.25 \%$ sodium fluorescein. The tip is advanced to approximate the cornea. The biprism splits the circle of contact into two semicircles. When the inner margins of these semicirculars touch, a $3.06 \mathrm{~mm}$ diameter circle of cornea is applanated. The IOP (in $\mathrm{mm} \mathrm{Hg}$ ) equals the flattening force (in gms) multiplied by ten. Sources of Error:

- Wide, blurred mires and vertical misalignment results in false high readings. 
- Corneal astigmatism: results in $1 \mathrm{~mm}$ error for every three diopters, underestimating for with the rule and overestimating for against the rule astigmatism.

- Corneal thickness: Thin corneas and corneas thickened by edema produce false low IOP readings while thick corneas due to increased collagen result in false high reading.

- Calibration should be done routinely (at least montly).

b) Perkins applanation tonometer: It uses the same biprism as the Goldmann tonometer. The light source is powered by battery. It is specially useful for infants, children and invalid patients who cannot sit at slitlamp.

c) Drager's applanation tonometer: It is similar to the Goldmann and Perkin's applanation accept that different biprism is used. This instrument is also portable and useful in similar situations as the Perkin's tonometer.

d) Non-contact tonometer (NCT): Introduced by Grolman in 1972, in airpuff tonometry, the applanating force is a column of air which is emitted with gradually increasing intensity. At the point of corneal flattening, the air column is shut off and the force at that moment is recorded and converted to $\mathrm{mm} \mathrm{Hg}$. The readings correlate well with Goldmann applanation tonometer [1]. Pulsair tonometer is a newer, portable handheld, non-contact tonometer which has a precision within $1 \mathrm{~mm}$ of $\mathrm{Hg}$ of the Goldmann appalanation tonometer.

Limitations of NCT:

- The time interval for an average NCT measurement is $1-3$ milli-second which is $1 / 500^{\text {th }}$ of one cardiac cycle. The measurements are random with respect to the phase of the cardiac cycle, so that ocular pulse becomes a significant variable i.e. it can't be averaged with some tonometers.

- Glaucomatous eyes have greater momentary fluctuations in IOP. Hence, it is recommended that a minimum of three readings within $3 \mathrm{~mm} \mathrm{Hg}$ to be taken and averaged as IOP.

e) Ocular response analyzer: The ocular response analyzer is a newer type of non-contact tonometer. The ocular response analyzer notes the moment of applanation, but the air column continues to emit with increasing intensity until cornea is indented. The force of air column then decreases until the cornea is once again at a point of applanation. The difference in the pressure at the two applanation points is a measure of corneal elasticity (hysteresis). Mathematical equations can be used to correct the applanation point for high and low elasticity [2].

f) Tonopen:

Principle- The tonometer applanates the cornea on contact using a plunger that moves within a sleeve. Applanation of the cornea moves this tip relative to the plunger and this movement is recorded as a continuous tracing following detection by a transducer. As the forces of corneal resistance are transferred to the sleeve, the applanation pressures concord with the IOP [3]. As the area of applanation of tonopen is smaller than GAT $\left(2.36 \mathrm{~mm}^{2}\right.$ Vs $\left.7.35 \mathrm{~mm}^{2}\right)$ therefore, theoretically the difference between applanating pressure and IOP is reduced due to reduced corneal resistance of a smaller contact area [4].

Procedure: The tonopen probe tip should be covered with a new ocu-film tip cover for each patient. After instilling a drop of anesthetic, the patient, sitting or supine, is asked to fixate on a point of reference. The instrument should be held like a pencil. To initiate an IOP measurement, the operating button is pressed and released once; and a brief display of [88888] will be seen as a self-test of LCD. When the tonopen is ready to measure, the LCD will display a double row of dashes [===], the green LED will light, and the device will beep. The sensor should be tapped very lightly and briefly on the corneal surface.

Some studies have found measurements taken by tonopen to be as accurate as the Goldmann applanation tonometer [5,6]. Several authors have demonstrated that tonopen tends to overestimate low IOPs and underestimate high IOPs $[7,8,9,10]$. Others have concluded that there is no such variation $[11,12]$.

\section{Indentation tonometery:}

Principle- The cornea is indented in the shape of a truncated cone by standard weights $(5.5 \mathrm{gms}$, $7.5 \mathrm{gms}, 10 \mathrm{gms}, 15 \mathrm{gms}$ ) which are applied via a plunger which moves freely within a shaft in the footplate .

Technique: The patient is laid supine with the cornea anesthetized. The fingers of the examiner spread the eyelid without putting pressure on the globe while patient is asked to fixate at a distant object. The needle on the tonometer scale oscillates with the ocular pulse and the mid-point of the excursion is used as the scale reading (Friedenwald table). If the value of the scale reading is not greater than four units, additional weights are added. Sources of error:

- Errors inherent to the instrument- Due to difference in weights of different parts of tonometer; difference in size, shape and curvature of footplate; friction arising in movement of plunger and pointer of the scale.

- Errors due to contraction of the extra-ocular muscles-

- Errors due to accommodation. 
- Errors due to variation of corneal curvature: steep and thick corneas- record false high IOP.

- Errors due to high scleral/ ocular rigidity.

- Observer's errors.

- Mose's effect: False high IOP recorded if the cornea gets sucked into the space between plunger and hole in footplate.

Limitations:

- In cases of high ocular rigidity, the conversion tables overestimates IOP and in cases of low ocular rigidity, the conversion tables underestimates IOP.

- Corneal variables: False high IOP is obtained with thick corneas or very steep corneas. Measurements are unreliable in significant corneal pathologies.

\section{Rebound tonometry}

The first prototypes of rebound type tonometer were developed by Antti Kontiola in 1994. The newest version of tonometer is ICare device. A $1.8 \mathrm{~mm}$ diameter plastic ball on a stainless steel wire is held in place by an electromagnetic field in a haldheld battery-powered device. When a button is pushed, a spring drives the wire and ball forward rapidly. When the ball hits the cornea, the ball and wire decelerates; the deceleration is more rapid if the IOP is high and slower if the IOP is low. The speed of the deceleration is measured and is converted by the device into IOP. No anesthetic is necessary. It shows good agreement with Goldmann and tonopen readings [1]. IOP measurements obtained with this tonometer have also shown to be influenced by corneal thickness, with higher IOP readings with thicker corneas [13, 14]. This tonometer has been shown to be affected by other biomechanical properties of the cornea, including corneal hysteresis and corneal resistance factor $[15$, $16]$.

\section{Pascal Dynamic Contour tonometry}

Pascal DCT is a slit lamp mounted digital tonometer. It makes use of pressure sensors which are accurate and stable devices for measuring the IOP non-invariably. The contour matched tonometer tip has a concave surface which allows the cornea to assume the shape which is naturally taken up when pressure on both sided of the cornea are equal. Contour matching is independent of contact diameter and appositional force. It converts the detected pressure digitally into a numeric result with 12 bit numerical precision and free from operator bias. There is no need for additional pachymetry and conversion of tonometer estimate. In conclusion, Pascal tonometry is a precise, contour and dynamic tonometry which has been introduced with the idea to overcome the factors related to corneal thickness and biomechanics. DCT can also be used to measure the ocular pulse amplitude. Disposable covers are used for each measurement and the digital display provides a QW value which assesses the quality of measurement $[13,17]$.

\section{Non-corneal and trans-palpebral tonometry:}

Diaton tonometer - Trans-palpebral tonometry refers to methods of measuring intra-ocular pressure through the eyelid. The Diaton non-corneal tonometer calculates pressure by measuring the response of a free falling rod, as it rebounds against the tarsal plate of the eyelid through the sclera. The patient is positioned so that the tip of the device and lid are overlying sclera. Non-corneal and trans-palpebral tonometry does not involves contact with the cornea and does not require topical anesthetic during routine use. The tonometer is not a substitute or alternative for Goldmann tonometry but may be helpful as a screening tool $[18,19]$. It is independent of corneal biomechanics such as corneal thickness, other biometric properties and intra-ocular pressure can be obtained in patients wearing contact lenses. In addition, non-invasive tonometry technique eliminates risk of infecting or scratching the cornea and it is perfect for use in remote locations as well as for mass glaucoma screenings.

\section{OCT tonometery}

Non-contact tonometry using optical coherence tomography (OCT) is currently under development. Like many other forms of tonometry, this methodology relies on a force being applied to the cornea and simultaneous measurement of the corneal reaction. In the case of OCT tonometry, the force applied to the cornea can be air pressure in the form of a high pressure jet (like air-puff tonometry), a shock or acoustic wave, or low pressure air using air pumped into a sealed chamber around the eye (like a snorkeling or scuba mask). The OCT device is used to measure changes in the curvature of the cornea or movement of the apical corneal interface. This deflection can be compared to the pressure measured around the eye and corrected for corneal thickness and potentially, corneal hysteresis. 


\section{References:}

[1] Bhan A, Browning A.C., Shah S. et al. Effect of corneal thickness on intraocular pressure measurements with the pneumotonometer, Goldmann applanation tonometer, and tonopen. Invest Ophthalmol Vis Sci 2002; 43(5): $1389-92$.

[2] Stamper R. A History of Intraocular Pressure and its Measurement. Optom Vis Sci 2011; 88(1): E16-28.

[3] Mackay RS, Marg E: Fast, automatic electronic tonometer based on an exact theory. Acta Ophthalmol 1959;37: 495-507.

[4] Orssengo G, Pye DC: Determination of the true intraocular pressure and modulus of elasticity of the human corena in vivo. Bull math Biol 1999;61:551-572.

[5] Denis P, Nordmann JP, Bertin V, Gayraud JM et al. Evaluation of the tonopen 2 and the XPert noncontact tonometers in cataract surgery. Ophthalmologica 1993;207:155-61

[6] Eisenberg DL, Sherman BG, McKeown CA, Schuman JS. Tonometry in adults and children. A manometric evaluation of pneumatonometry, applanation, and TonoPen in vitro and in vivo. Ophthalmology. 1998 ; 105:1173-81.

[7] Kao SF, Lichter PR, Bergstrom TJ, Rowe S. Clinical comparison of the oculab tonopen to the goldmann applanation tonometer. Ophthalmol 1987;91:1541-44

[8] Frenkel RP, Hong J, Shin DH. comparison of the tonopen to the Goldmann applanation tonometer. Arch Ophthalmol 1988; 106:750-53.

[9] Geyer O, Mayron Y,Loewenstien A,et al.Tonopen tonometry in normal and post keratopalsty eyes. Br J Ophthalmol 1992; 76:53840 .

[10] Lester M, Mermoud A, Achache F, Roy S. New Tonopen XL: comparison with the Goldmann tonometer. Eye. 2001;15(Pt 1): 52-8.

[11] Tonnu P-A Tonnu,1 T Ho,1 K Sharma,1 E White,1 C Bunce,2 and D Garway-Heath1 A comparison of four methods of tonometry: method agreement and interobserver variability. Br J Ophthalmol. 2005; 89: 847-850.

[12] Bafa M, Lambrinakis I, Dayan M, Birch M. Clinical comparison of the measurement of the IOP with the ocular blood flow tonometer, the Tonopen XL and the Goldmann applanation tonometer. Acta Ophthalmol Scand. 2001;79:15-8.

[13] Pakrou N, Gray T, Mills R, et al. Clinical comparison of the Icare tonometer and Goldmann applanation tonometry. J Glaucoma. 2008 Jan-Feb;17(1):43-47.

[14] Poostchi A, Mitchell R, Nicholas S, et al. The Icare rebound tonometer: comparisons with Goldmann tonometry, and influence of central corneal thickness. Clin Experiment Ophthalmol. 2009 Sep;37:687-691.

[15] Chi ,WS, Lam A, Chen D, et al. The influence of corneal properties on rebound tonometry. Ophthalmology 2008;115:80-84.

[16] Jorge Jm, Gonzalez-Meijome JM, Queiros A, et al. Correlations between corneal biomechanical properties measured with the ocular response analyzer and ICare rebound tonometry. J Glaucoma. 2008;17:442-448.

[17] Kniestedt C, Lin S, Choe J, et al. Clinical comparison of contour and applanaion tonometry and their relationship to pachymetry. Arch Ophthalmol 2005; 123: 1532-1537.

[18] Li Y, Shi J, Duan X, Fan F. Transpalpebral measurement of intraocular pressure using the Diaton tonometer versus standard Goldmann applanation tonometry. Graefes Arch Clin Exp Ophthalmol. 2010 Dec; 248(12):1765-70.

[19] Troost A, Yun SH, Specht K, Krummenauer F, Schwenn O. "Transpalpebral tonometry: reliability and comparison with Goldmann applanation tonometry and palpation in healthy volunteers." Br J Ophthalmol. 2005 Mar; 89(3):280-3. 